\title{
Quality of Service Optimization in Realm of Green Monitoring using Broad Area Sensor Network (BASN)
}

\author{
Ajeet kumar ${ }^{1}$, Pankaj kumar ${ }^{2}$, Ashwini Singh ${ }^{3}$,Dr. Ritesh Kumar Mishra ${ }^{4}$ \\ ${ }^{\prime}$ (Department of ECE, National Institute of Technology, India) \\ ${ }^{2}$ (Department of ECE, National Institute of Technology, India) \\ 3 (Department of ECE, National Institute of Technology, India) \\ ${ }^{3}$ (Asst. Prof. Department of ECE, National Institute of Technology, India)
}

\begin{abstract}
A Wireless Sensor Network(WSN) contains short range energy limited terminals/nodes in which multiple nodes participates one by one to transfer data from source node to Base station. Each node appends some amount of delay which degrades the network performance as the jittered behaviour of network may not be allowed in some applications. Again the faulty nature of tandem nodes may create some severe routing scenarios. Concept of Broad Area Sensor Network (BASN) incorporates a real time communication between sensor node and Base station, which helps to optimize the Quality of Service (QoS). Our goal is to do extensive simulation on performance analysis of such sensor networks and its optimization.
\end{abstract}

Keywords - WSN, energy limited terminals, jitter, Quality of service(QoS)

\section{Introduction}

Wireless Sensor Network (WSN) is a set of individual nodes that are able to interact with the environment by sensing or controlling physical parameters. The evolution of small form-factor, single-chip wireless transmitter and receiver has enabled many new previously unimaginable forms of connectivity which is gift for the emerging wireless world. One such area which has benefited from low-cost miniature radios is the ability to not only acquire information from micro-sensors but also transmit this data in real time over a wireless link. For the past few years researchers are trying to explore new circuits topology for sensor node, different network methods, different radio architecture and approaches to energy scavenging which allow autonomous operation of wireless sensor network for long time [1]. At present the WSN Generally uses MESH topology of sensor nodes which individually constraint to small coverage area due to limited internal energy of fixed small size sensor nodes.

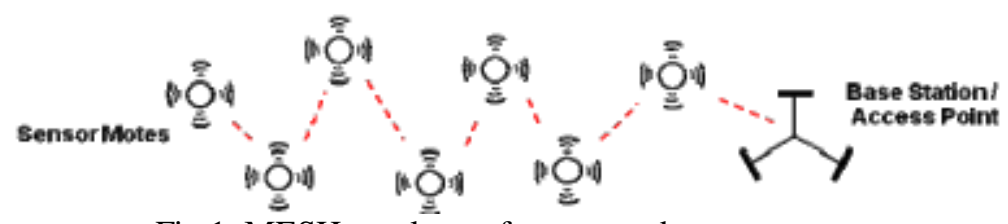

Fig.1 MESH topology of sensor nodes

previous analysis based on the well-known path loss characteristics of isotropic radiation using (1), has shown that an optimal distance between individual nodes in a collaborative mesh system theoretically yields the lowest energy consumption per bit, when transmitting a given distance " $d$ " [1].

$$
P_{r}=P_{t} \cdot G_{t} \cdot G_{r} \cdot\left(\frac{1}{4 \pi d f}\right)^{2}
$$

In such type of network, periodically each node must "wake up" to assess whether data from adjacent nodes must be routed through said node. This requires additional "receive wakeup" energy to allow network synchronization and routing .Also in this case router nodes participates one by one to transfer data from source node to Base station. Due to congestion in path and transmit and receive time each node appends some amount of delay which degrades the network performance. The optimal energy usage in mesh networks assumes each node is ideally positioned relative to adjacent sensor nodes. This may not be practical, particularly for applications where the individual sensors nodes are deployed from an airplane or mobile vehicle with the resulting final position of each sensor mote, relative to other nodes, being random [2]. This is particularly true in many defense applications where the sensors are relatively remote and deployed randomly; an example being barrier coverage applications. The power advantages associated with mesh networks are further challenged upon initial deployment by the complexity associated with "network self-assembly". In such cases, deployment of a large mesh network to acquire information in remote locations would require numerous nodes collecting 
redundant data. Lastly, there are a plethora of envisioned applications which would require sensor mobility, further complicating the routing of data in a mesh network.

This generally used sensor network topology is suitable for many applications, but for applications like military operation or other which strictly needed real time data transfer, this is not an efficient choice. Our paper contains an approach for different WSN scenario which contains real time data transfer between sensor nodes and Base station. In this paper, an approach to sensor network communication which more closely resembles characteristics of Broad Area Network (BAN) is proposed as an alternative to mesh systems. Specifically, this work seeks to explore communication of a sensor node to a base-station several kilometers away.

\section{Wireless BSN System}

A key aspect of the Wireless Broad Sensor Network (BSN) is the realization of small form-factor wireless transceivers which utilize conventional energy scavenging devices, such as solar cells, and have the ability to transmit several kilometers with modulation methods compatible with communication on either standards-based wireless networks or custom designed systems. If future sensor motes could transmit data over distances commensurate with cellular communication, the potential exists to leverage available infrastructure and thus provide coverage for virtually all urban and suburban locations worldwide. Moreover, potential multimode solutions would further expand network access through standards based systems such as WiFi and Bluetooth. With sufficient range enabled through new transceiver architectures, these wireless devices could be made to communicate with access points found either on Unmanned Ariel Vehicles (UAVs) or Satellite stations. Such a transceiver will be realized through a combination of new wireless technologies which specifically emphasize small form-factor, high integration, low-cost transceivers which communicate over long distances. New methods of signal modulation, antennas customized for high gain when placed close to a ground plane, customized transceiver architectures which optimize energy efficiency rather than power efficiency and new methods of energy scavenging will all play a roll towards realizing long-distance wireless sensor communication.

Wireless BSN system is ideally suited for remote sensor applications or those requiring low spatial density. Examples could include environmental monitoring [3] or homeland security applications - such as "tripwires" - to sense chemical, biological, or nuclear material [4].

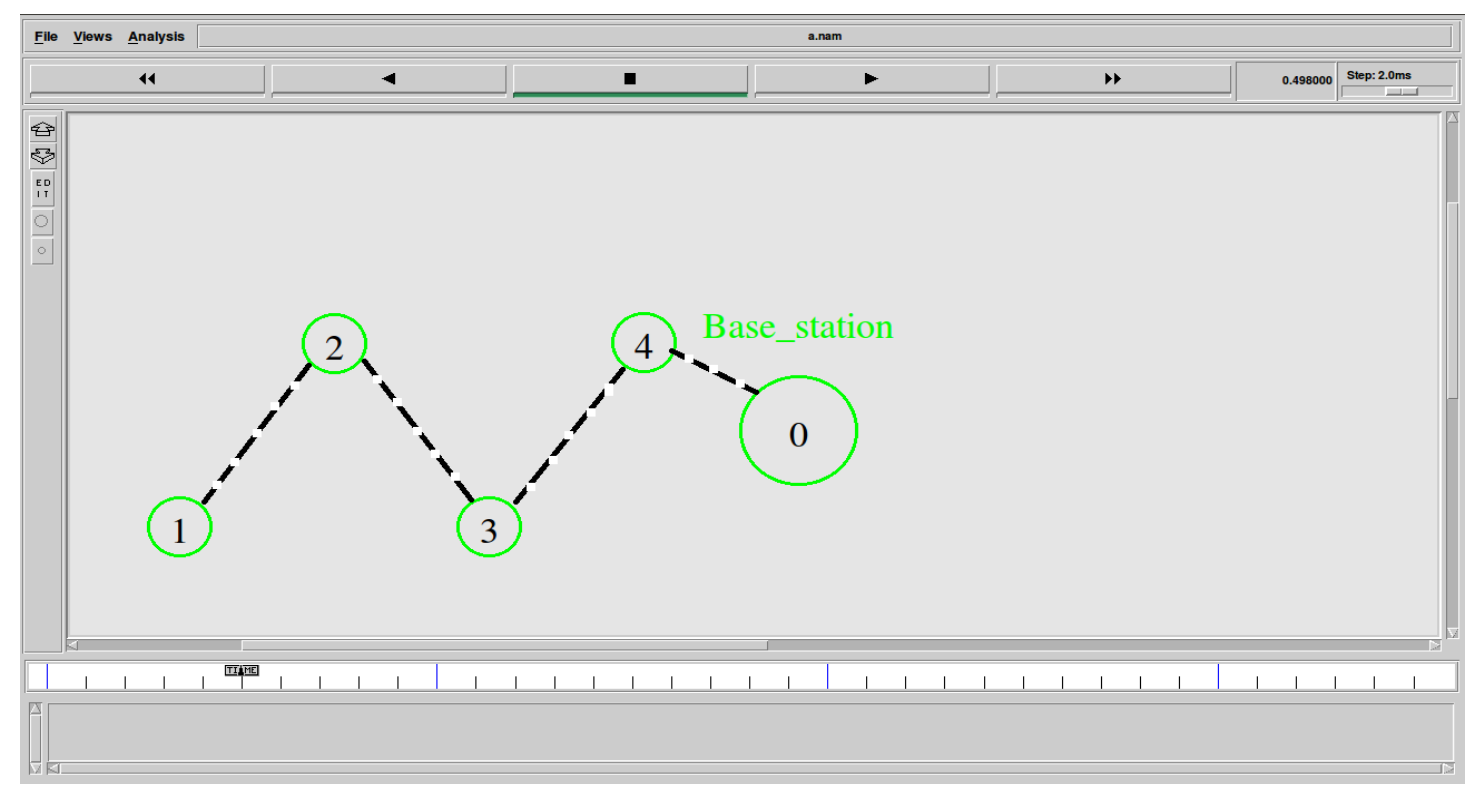

Fig. 2 MESH topology data transfer ( $\mathrm{n} 1$ is source node, base station is destination node) 


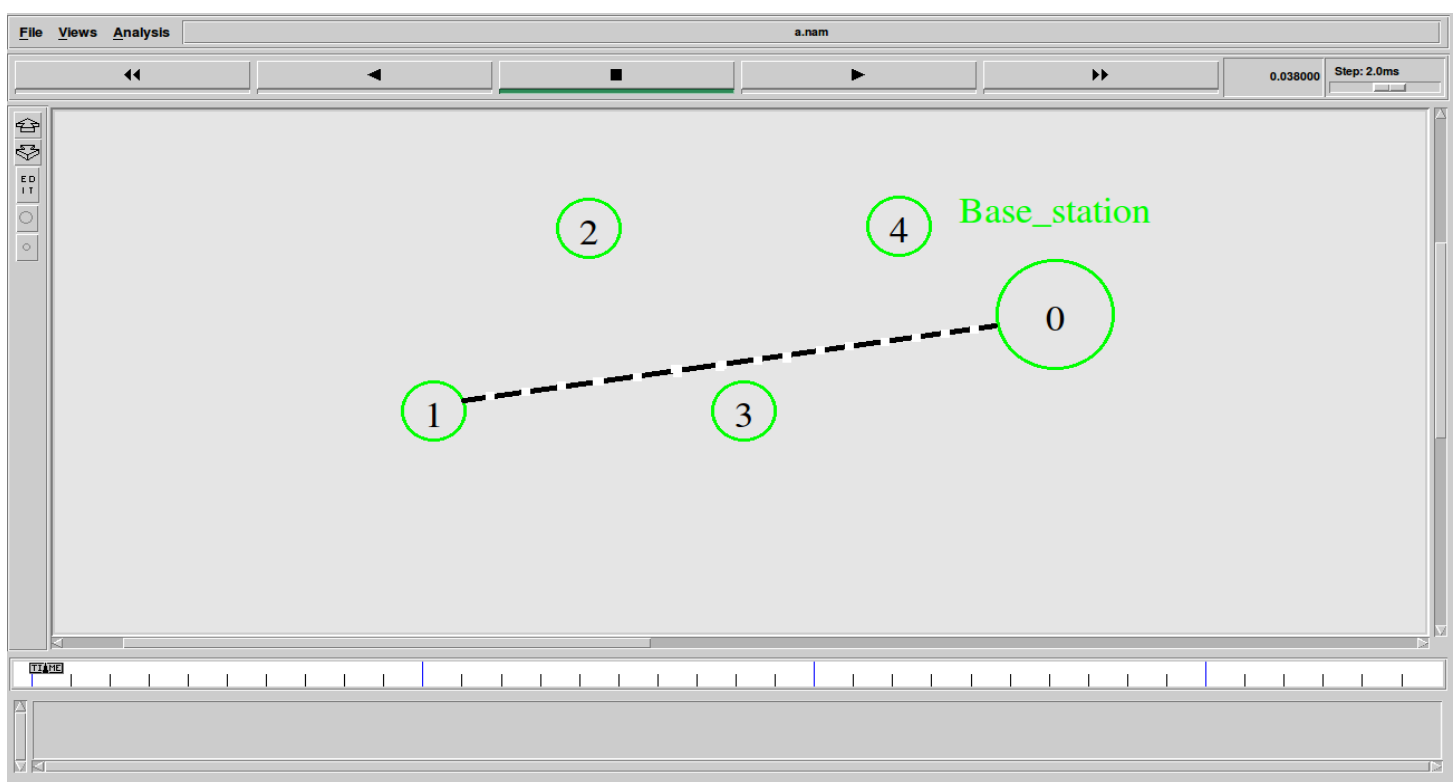

Fig. 3 Wireless BSN network topology(n1 is source node, Base station is Destination )

Fig. 2 represents the generally used MESH topology WSN in which data sensed by " 1 " transferred to "Base station" through $\mathrm{n} 2, \mathrm{n} 3$ and $\mathrm{n} 4$. So it requires additional receiving and transmission of $\mathrm{n} 2, \mathrm{n} 3$ and $\mathrm{n} 4$.

Fig. 3 represents Wireless BSN system of 4 sensor nodes in which data sensed by " 1 " is directly transferred to "Base station", means real time communication.

Now we can extent this process as, suppose $\mathrm{n} 2$ sense and want to transfer data to Base station. According to conventional WSN (Fig. 2) scenario data goes as $n 2 \Rightarrow n 3 \Rightarrow n 4 \Rightarrow$ Base_station. So extra delay and extra power loss encountered by routing node $\mathrm{n} 3$ and $\mathrm{n} 4$. According to the proposed scenario data will directly transfer from $\mathrm{n} 2$ to Base_station. Same for $\mathrm{n} 3$ and $\mathrm{n} 4$. In case of $\mathrm{n} 4$ node the delay and power loss will be same for both scenario. One particular remote-sensing application that has garnered research attention is that of reliable barrier coverage [2]. The ability to surreptitiously detect vehicle or troop movement can provide significant advantages in applications on both home and hostile soil. Depending on the area to be covered, the only means of barrier installation may be by airdrop. This coupled with acquisition of sensor data by UAVs is a proposed new scenario for recovering data in the field. If a short range wireless network is utilized, issues with receive wake up energy, node placement, and self-assembly may limit the practical area of barrier coverage. Since Wireless BSN system contains long-range wireless sensors, barrier coverage can be utilized in extremely remote areas.

\section{Feasibility of BSN}

A key aspect for the feasibility of Wireless BSN system is the ability to acquire enough energy in the sensor mote to allow a transmit (TX) burst at high output powers .A case study of GSM system gives out the result given in Table.1

\begin{tabular}{|l|l|l|l|l|}
\hline & $\begin{array}{l}\text { Base station } \\
\text { transmit }\end{array}$ & $\begin{array}{l}\text { Handset } \\
\text { transmit }\end{array}$ & $\begin{array}{l}\text { Peak handset } \\
\text { power }\end{array}$ & $\begin{array}{l}\text { Used in the UK Primarily } \\
\text { by }\end{array}$ \\
\hline GSM 900 & $935-960 \mathrm{MHz}$ & $\begin{array}{l}890-915 \\
\text { MHz }\end{array}$ & 2 Watt & BT Cellnet and Vodafone \\
\hline GSM 1800 & $1805-1880 \mathrm{MHz}$ & $\begin{array}{l}1710-1785 \\
\text { MHz }\end{array}$ & 1Watt & Orange and One2 One \\
\hline
\end{tabular}

This study focuses on the upbanded version of GSM-PCS 1900 , at $1.9 \mathrm{GHz}$, with a maximum output power of 1Watt. The question becomes, is it possible to transmit a sustained burst of $+30 \mathrm{dBm}$ (1Watt) for a duration of one GSM timeslot $(577 \mu \mathrm{s})$ using optimized circuit containing solar cells and supercapacitors as a energy generating and energy storing device respectively? 


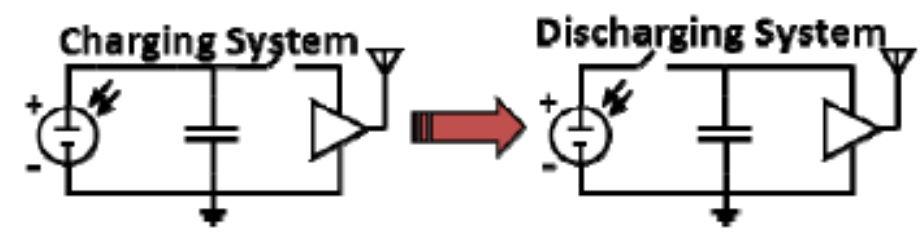

Fig. 4 optimized circuit diagram containing solar cell, super capacitor and power amplifier.

\subsection{Evaluation using mathematics}

We have done comparison between the energy generated by Fig.4 circuit and the energy required for transmission in GSM 1800 using mathematical expressions.

$$
\frac{1}{2} C \Delta V^{2}=\mathrm{P} * \mathrm{Ts} * \mathrm{~N}
$$

Left hand side of expression (2) represents the energy generated and stored by circuit containing super capacitor and solar cell.

Where $\mathrm{V}$ is the voltage generated by solar cell changes from $1 \mathrm{v}$ to $2.5 \mathrm{v}$ approx.(Refer Table 2 ) So $\triangle \mathrm{V}=1.5$,

$\mathrm{C}=$ capacitance value

Table 2

\begin{tabular}{|l|c|c|}
\hline \multicolumn{1}{|c|}{ Power Source } & Power Density $\left(\mu \mathrm{WW} / \mathrm{cm}^{3}\right)$ & Lifetime \\
\hline Lithium Battery & 100 & $1 \mathrm{yr}$ \\
\hline Solar Cell & $10-15000$ & $\infty$ \\
\hline Air Flow & 380 & $\infty$ \\
\hline Temperature Gradient & 50 & $\infty$ \\
\hline
\end{tabular}

Right hand side of equation (2) is the total energy required for transmission in GSM 1800 for 1 time slote. Power (p)=power needed for transmission for GSM 1800(Refer Table 1)

$\mathrm{P}=1$ watt

Time slot $(\mathrm{Ts})=577 \mu \mathrm{s}$

$\mathrm{N}=$ no. of timeslot, Considering 1 time slot so $\mathrm{N}=1$

By putting these values we can find the value of capacitance $(C)$, which is approximately equals to $0.512 \mathrm{mF}$

\subsection{Evaluation using graph}

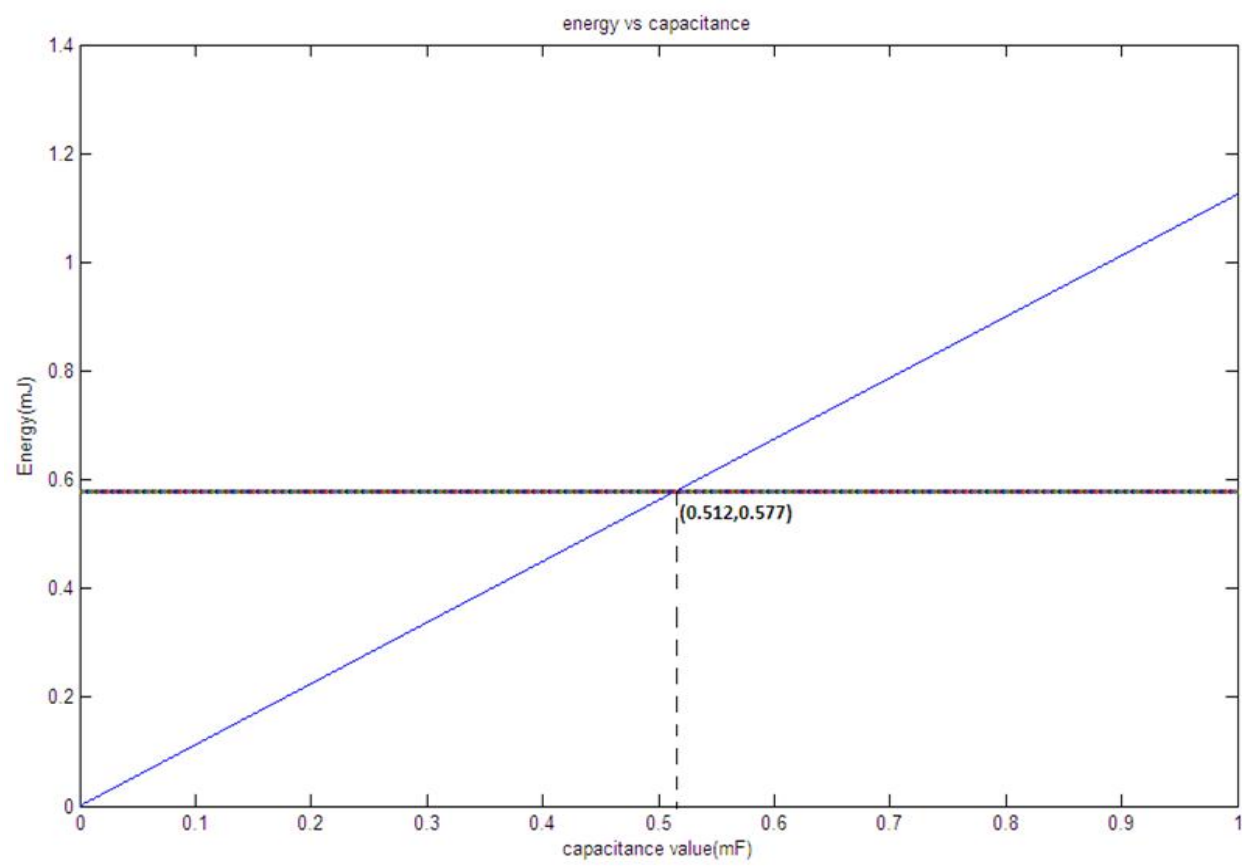

www.iosrjournals.org 
So it is possible to use Broad Sensor Network using given circuit of super capacitor having capacitance value approximately equals to $0.512 \mathrm{mF}$

\section{Remaining Energy comparison using ns2:-}

In previous sections we have done surveys and evaluation on existence possibility of BSN and have got positive result. Now its time to implement it .

Suppose data is sensed by node $n 1$ and passed through $n 2, n 3$ and $n 4$ to Base station. In case of MESH topologyIt requires 4 transmitting and 4 receiving process [Fig. 2]. In case of BSN topology it requires one transmitting and one receiving process. As we know other energy factor like ideal or sleep power not majorly affect the whole energy scenario so MESH topology will take more power then BSN topology.

We have done an ns2 simulation of wireless network scenario containing four sensor nodes and one Base station using existed MESH topology and Wireless Broad Area Sensor Network separately and at the last of simulation compared the remaining available energy level.

Sample configuration used in our program

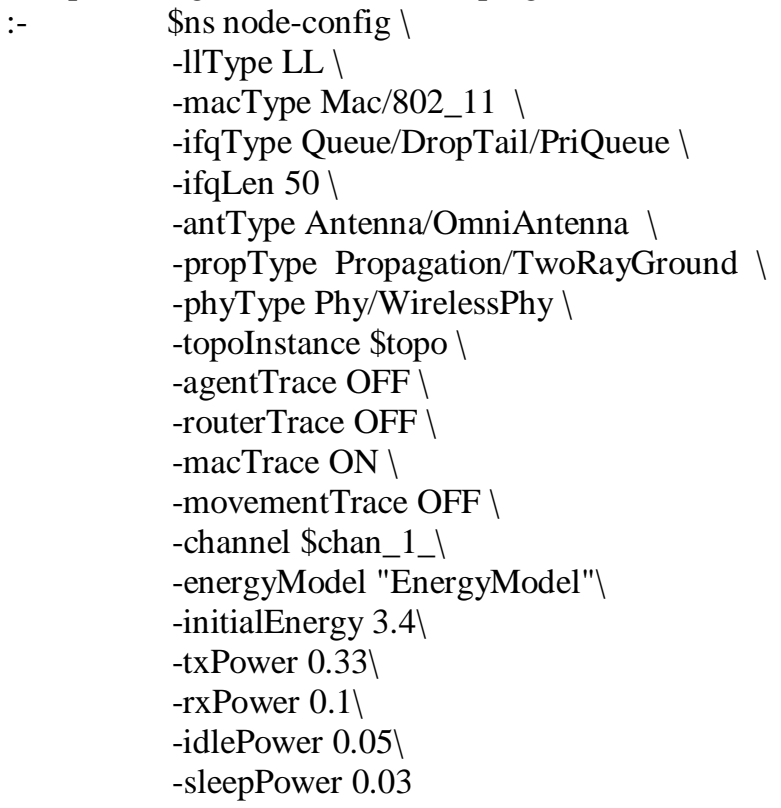

4.1 Using MESH topology simulation result:-

\begin{tabular}{|c|l|l|l|}
\hline Node & $\begin{array}{c}\text { Initial } \\
\text { Energy(J) }\end{array}$ & $\begin{array}{l}\text { Energy after } \\
\text { simulation }\end{array}$ & $\begin{array}{l}\text { Total energy } \\
\text { consumed }\end{array}$ \\
\hline $\mathbf{n ~ 1 ~}$ & 3.400000 & 3.222223 & 0.177777 \\
\hline $\mathbf{n} \mathbf{2}$ & 3.400000 & 3.200855 & 0.199145 \\
\hline $\mathbf{n}$ 3 & 3.400000 & 3.183228 & 0.216772 \\
\hline n 4 & 3.400000 & 3.168332 & 0.231668 \\
\hline Base_station & 3.400000 & 3.201656 & 0.198344 \\
\hline
\end{tabular}

Total energy loss $=1.023706$

\subsection{Using wireless BSN system topology:-}

\begin{tabular}{|c|l|l|l|}
\hline Node & Initial Energy & $\begin{array}{l}\text { Energy after } \\
\text { simulation }\end{array}$ & $\begin{array}{l}\text { Total energy } \\
\text { consumed }\end{array}$ \\
\hline $\mathbf{n}$ 1 & 3.400000 & 3.224075 & 0.175925 \\
\hline $\mathbf{n} \mathbf{2}$ & 3.400000 & 3.214133 & 0.185867 \\
\hline n 3 & 3.400000 & 3.203635 & 0.196365 \\
\hline Base_station & 3.400000 & 3.193167 & 0.206833 \\
\hline
\end{tabular}

Total energy loss $=0.966180$ 


\section{Conclusion}

A new concept for long-range sensor communication was presented. Although many questions and challenges remain for the realization of Wireless Broad Sensor Network (BSN) System, some aspects appear feasible. In particular, this paper presented the mathematical and graphical analysis on feasibility of BSN and ns2 simulation on implementation of BSN. Other performance parameters like "Time Delay" should also be analyzed using suitable tools.

\section{REFERENCES}

[1] B. Cook, S. Lanzeisera, and K. Pister, "SoC Issues for RF Smart Dust", Proc. of the IEEE, vol. 94, pp. 117-1196, June 2006.

[2] A. Saipulla, B. Liu and J. Wang, "Barrier Coverage with Airdropped Wireless Sensors", Proc. of Miltary Comms Conf, pp. 1-7, 2008.

[3] K. Martinez, J.K. Hart, and R. Ong, "Environmental Sensor Networks", Computer,Vol.37, Iss.8, August 2004.

[4] R. Nemzek, J. Dreicer, and D. Torney, "Distributed Sensr Networks for Detection of Mobile Radioactive Sources," IEEE Nuclear Science Symposium Conference, Vol. 3, pp. 1462-1467, Oct. 19-25 2003. 